\title{
DIFUSÃo MULTICOMPONENTE DURANTE A SALGA MISTA DE QUEIJO PRATO
}

\author{
Evandro BONA ; Dionísio BORSATO ; Rui Sérgio S. F. da SILVA ; Luiz Henry M. e SILVA
}

\section{RESUMO}

A relação entre o $\mathrm{NaCl}$ e a hipertensão arterial tem estimulado a sua substituição parcial por $\mathrm{KCl}$ em queijos. Entretanto, para manter a qualidade final do produto, é de suma importância assegurar uma proporção adequada de $\mathrm{NaCl} / \mathrm{KCl}$, bem como a distribuição homogênea destes sais. Os modelos que estimam a concentração salina, portanto, são valiosas ferramentas para o controle de qualidade. Para simular a difusão multicomponente durante a salga em salmoura estática, foi proposto um modelo unidimensional, baseado na solução analítica da $2^{a}$ lei de Fick generalizada para dois solutos. A solução analítica, implementada através de um algoritmo computacional, permitiu o ajuste dos coeficientes de difusão e a avaliação da resistência externa. Os resultados da simulação obtidos apresentaram boa concordância com os valores experimentais (desvio de 2,7\% para o $\mathrm{NaCl}$ e $6,6 \%$ para o $\mathrm{KCl}$ ), validando a capacidade preditiva do modelo proposto. Além disso, a simulação pode permitir um ajuste de ganhos de produtividade (redução do tempo de salga) através da estimativa do tempo de salga para teores salinos desejados.

Palavras-chave: coeficientes de difusão cruzados; salmoura estática; alimentos para fins especiais.

\section{SUMMARY}

MULTICOMPONENT DIFFUSION DURING SIMULTANEOUS BRINING OF PRATO BRAZILIAN CHEESE. The relation between $\mathrm{NaCl}$ and arterial hypertension has stimulated a partial substitution for $\mathrm{KCl}$ on cheese. However, it is very important to guarantee an adequate proportion of $\mathrm{NaCl} / \mathrm{KCl}$, and also the homogeneous distribution of these salts, to maintain the final quality of the product. Therefore, the models that calculate the salty concentration are useful tools to control the quality. In this study an analytic resolution of Fick's 2 law, generalized for two salts, was used to simulate the salting in stationary brine. The analytic resolution, carried out through a computer program, allowed an adjustment of the diffusion coefficients and to evaluate the external resistance. The results calculated show a close comparison of the experimental values (an deviation of $2.7 \%$ to $\mathrm{NaCl}$ and $6.6 \%$ to $\mathrm{KCl}$ ), validating the proposed model. The simulation can be used to adjust productivity (reducing the salting time) through the salting-time calculation for a required saltiness.

Keywords: cross diffusion coefficients; stationary brine; and dietary foods.

\section{1 - INTRODUÇÃO}

Por serem uma importante fonte de proteínas, fósforo, cálcio e alguns micronutrientes, os queijos estão relacionados entre os alimentos considerados nutricionalmente importante [10]. Entretanto, algumas pessoas têm evitado seu consumo com o objetivo de reduzir a ingestão de cloreto de sódio e, conseqüentemente, amenizar os problemas relacionados à hipertensão [17]. Isto porque, o consumo de sódio é um dos principais fatores que, comprovadamente [14], causam o aumento da pressão arterial.

Vários substitutos para o cloreto de sódio têm sido estudados e entre eles se destaca o cloreto de potássio, por apresentar propriedades fisicas semelhantes $[8,18,19$, $24,33]$. O potássio apresenta um efeito diurético que aumenta a excreção dos íons sódio pelos rins, reduzindo a

Recebido para publicação em 10/09/2004. Aceito para publicação em 04/05/2005(001403).

Departamento de Quimica, Universidade Estadual de Londrina. C.P.: 6001. CEP: 86051-990, Londrina-Paraná. E-mail: bona@uel.br, dborsato@uel.br:

Departamento de Tecnologia de Alimentos e Medicamentos, Universidade Estadual de Londrina, C.P.: 6001. CEP: 86051-990, Londrina-Paraná.E-mail: ruisergio@uel.br

Faculdade de Apucarana, Apucarana-Paraná. E-mail: lhm.silva@fap.com.br

A quem a correspondência deve ser enviada. pressão arterial [1]. Segundo HE \& MACGREGOR [14] uma ingestão de $64 \mathrm{mmol} /$ dia reduz a pressão de 1,4 a $2,7 \mathrm{mmHg}$ em pacientes hipertensos. Além disso, o potássio diminui o risco de acidente vascular cerebral e reduz a excreção de cálcio pelos rins, evitando a formação de calcificações nos rins e a desmineralização dos ossos [15]. A substituição completa do $\mathrm{NaCl}$ pelo $\mathrm{KCl}$ não é recomendada, devido ao sabor amargo conferido ao produto, o que o torna pouco aceitável $[8,19,28]$. Contudo, a substituição de até $30 \%$ do cloreto de sódio pelo cloreto de potássio não apresentou diferenças sensoriais, fisicoquímicas ou microbiológicas significativas $(p<0,05)$ em relação ao queijo prato salgado apenas com cloreto de sódio [28].

Dos vários processos de salga, a imersão em salmoura é o mais utilizado. Este processo consiste na imersão dos queijos em uma solução de cloreto de sódio com concentração mantida constante. O sal, além de conferir sabor à massa, intervém na dessoragem do queijo, na formação da casca, no desenvolvimento microbiano, influindo ainda na textura do produto por dissolver parte da caseína [11]. Portanto, uma concentração média de sal, bem como a sua distribuição uniforme, são fatores importantes na maturação de queijos [13]. Conseqüentemente, os modelos que estimam a concentração salina como resultado das condições de processo poderão ser úteis quando mais de um componente for considerado na salga. 
Os aspectos teóricos do processo de difusão do cloreto de sódio no queijo têm sido estudados por vários autores [12, 23, 27, 33]. Muitos modelos de perda de água e ganho de soluto estão baseados na hipótese de que a transferência de massa pode ser descrita pelas leis de difusão de Fick [4, 30]. Quando vários solutos se difundem, simultaneamente, assume-se uma lei generalizada de Fick $[5,26]$. Neste caso, além do coeficiente de difusão principal de cada soluto, em relação ao seu próprio gradiente de concentração, são incluídos os coeficientes de difusão cruzados, que acoplam a influência de um soluto no fluxo do outro soluto. Geralmente, para solutos altamente dissociáveis (como o $\mathrm{NaCl}$ e $\mathrm{KCl}$ ) os termos cruzados são de ordem de grandeza inferior aos termos principais [12, 20, 25, 32].

O objetivo deste trabalho foi modelar e simular o processo de difusão multicomponente ocorrido durante a salga de queijo prato em salmoura estática, através de um modelo analítico fundamentado na $2^{\mathrm{a}}$ lei de Fick generalizada para dois solutos.

\section{2 - MATERIAL E MÉTODOS}

\section{1 - Procedimento experimental}

Sete amostras de queijo (QUEIJO PRATO LANCHE DI CARLO, Laticinios Campina Alta, Manoel Ribas PR), cortadas em formato hexaédrico com $12 \mathrm{~cm}$ (eixo Z) $\mathrm{x}$ $10 \mathrm{~cm}$ (eixo $\mathrm{Y}$ ) x 4cm (eixo X), foram mantidas durante 11 horas em salmoura estática a $10 \pm 1^{\circ} \mathrm{C}$. A salmoura foi preparada conforme FURTADO [10], que sugere uma concentração salina em torno de $20 \%(\mathrm{~m} / \mathrm{m})$ e $\mathrm{pH}$ médio de 5,5 ajustado com ácido clorídrico. Cloreto de cálcio foi adicionado, $0,5 \%(\mathrm{~m} / \mathrm{m})$, para evitar perda de $\mathrm{Ca}^{2+}$ nos queijos e, conseqüente, perda de textura. O volume de salmoura utilizado foi aproximadamente 3,5 vezes maior do que o volume de queijo, para garantir que a concentração da salmoura permanecesse constante durante o período de salga [12]. Antes do início da salga, foi retirada uma amostra da salmoura para a quantificação dos sais presentes, assim como ao final do período de salga. Para o estudo da difusão multicomponente foi utilizada uma proporção em massa entre os sais de $70 \%$ de $\mathrm{NaCl}$ para $30 \%$ de $\mathrm{KCl}$, conforme sugestão de RAPACCI [28]. Por apresentarem densidade inferior a da salmoura, os pedaços de queijo permaneceram flutuando durante o processo de salga. Logo, sobre a face voltada para cima existia apenas uma fina lâmina de salmoura. Essa face foi marcada, pois por hipótese, considerou-se que o ganho de sal poderia ser menor em relação à face voltada para baixo.

O procedimento de amostragem adotado, para o estudo da difusão multicomponente do $\mathrm{NaCl}$ e $\mathrm{KCl}$, consistiu na retirada de duas amostras cilindricas ao longo do eixo $\mathrm{X}$ de $1,5 \mathrm{~cm}$ de diâmetro e $4 \mathrm{~cm}$ de comprimento, posicionadas conforme descrito na Figura 1. Após a amostragem, a parte remanescente dos queijos foi reposta na salmoura apenas para manter constante a relação entre o volume de salmoura e o de queijo. Com a mesma finalidade, foi subtraída uma fração de salmoura corresponden- te ao volume das amostras cilindricas retiradas. Os intervalos de tempo de amostragem escolhidos foram de 1 , 2, 3, 5, 7, 9 e 11 horas de salga.

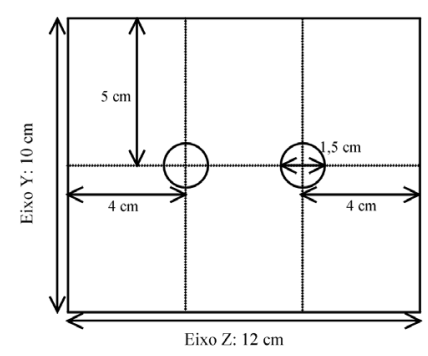

FIGURA 1 - Amostragem adotada para o estudo da difusão multicomponente

As dimensões escolhidas foram baseadas na penetração máxima estimada, aproximadamente $1,5 \mathrm{~cm}$, do $\mathrm{NaCl}$ durante o período de salga proposto [29]. A adoção do procedimento de amostragem teve como objetivo criar condições experimentais para que, nas amostras analisadas, a difusão pudesse ser considerada unidimensional $[12,23]$. Após a retirada, os cilindros foram cortados ao meio, resultando em quatro amostras com $2 \mathrm{~cm}$ de comprimento. Os cilindros pertencentes ao mesmo lado da face YZ (Figura 1) foram colocados em um cadinho numerado que foi pesado (balança AND, modelo HR-200) e colocado na estufa (FANEM RETILÍNEA) a $105^{\circ} \mathrm{C}$, para a determinação da umidade das amostras. Após a determinação da umidade, as amostras foram cuidadosamente carbonizadas em bico de Bunsen de modo a evitar o espalhamento e remoção da gordura e, então, colocadas em uma mufla (QUIMIS) à temperatura de $550^{\circ} \mathrm{C}$ durante 3 horas para serem incineradas. Posteriormente, no cadinho contendo a amostra, previamente resfriado, foram adicionados $15 \mathrm{~mL}$ de ácido clorídrico 0,5mol/L. Após a mistura do ácido com as cinzas, a solução resultante foi filtrada em papel filtro (QUANTY JP40 faixa branca). O resíduo foi lavado com ácido clorídrico 0,5mol/L em 3 porções de $20 \mathrm{~mL}$ cada vez. O filtrado foi recolhido em balão volumétrico de $100 \mathrm{~mL}$ e completado o volume com a adição de ácido clorídrico $0,5 \mathrm{~mol} / \mathrm{L}$. Os teores de sódio e potássio nas amostras foram determinados a partir de uma alíquota, convenientemente diluída, do filtrado obtido das cinzas. A solução resultante foi levada a um fotômetro de chama dotado de filtros interferenciais (CELM, modelo FC 280), segundo técnica descrita pela AOAC [2]. $O$ resultado fornecido foi convertido em concentração (mg/L) através de interpolação na curva padrão construida para cada um dos sais.

Uma amostra de queijo não submetida a salga foi utilizada para a quantificação dos teores iniciais de cloreto de sódio e cloreto de potássio. Parte desta amostra também foi utilizada na determinação de umidade (secagem em estufa até peso constante) e gordura (método Soxhlet). 


\section{2 - Modelagem analitica unidimensional da difusão multicomponente durante a salga}

Para a formulação do modelo analítico foram consideradas algumas hipóteses:

- A difusão multicomponente foi considerada unidimensional segundo o eixo $\mathrm{X}$, nas amostras analisadas.

- O sistema em estudo não é reacional, tendo em vista que as reações bioquímicas mais significativas ocorrem durante o período de maturação.

- O coeficiente de difusão ou difusividade mássica foi considerado constante em relação à concentração (independente do tempo e posição).

- A resistência externa é igual para os dois solutos, por serem estes compostos iônicos muito semelhantes.

- A contração da amostra foi desprezada, pois segundo a literatura [12] a variação de volume é mínima durante a salga de queijos.

O eixo X foi escolhido, pois como mencionado anteriormente, a amostragem realizada atentou para que a difusão fosse considerada unidimensional neste eixo. Ao processo de difusão multicomponente foi associado um comportamento Fickiano. A equação, portanto, que descreve a variação de concentração unidimensional do soluto (C) em uma placa plana finita é:

$$
\frac{\partial C}{\partial t}=D \frac{\partial^{2} C}{\partial x^{2}} \quad \text { para } \quad t>0 \quad \text { e }-R<x<R
$$

onde, $\pm \mathrm{R}(\mathrm{cm})$ é a distância até o centro do eixo analisado, D (cm / dia) é a matriz dos coeficientes de difusão, e $\mathrm{C}$ (g/100g de água no queijo) o vetor que contém a concentração dos dois solutos, representados respectivamente como:

$$
D=\left[\begin{array}{ll}
D_{11} & D_{12} \\
D_{21} & D_{22}
\end{array}\right] \quad \text { e } \quad C=\left\{\begin{array}{l}
C_{1} \\
C_{2}
\end{array}\right\}
$$

sendo, por convenção, $\mathrm{C}_{1}$ a concentração de $\mathrm{NaCl}$ e $\mathrm{C}_{2}$ a concentração de $\mathrm{KCl}$. Os símbolos $\mathrm{D}_{1,}$ e $\mathrm{D}_{22}$ representam os coeficientes de difusão principais de cada soluto em relação ao próprio gradiente de concentração. Os termos $\mathrm{D}_{12}$ e $\mathrm{D}_{21}$, referem-se aos coeficientes de difusão cruzados que acoplam o fluxo de um soluto com o gradiente do outro soluto. Foi utilizada como condição inicial $\mathrm{C}(\mathrm{x}, 0)=\mathrm{C}_{\mathrm{o}}$,

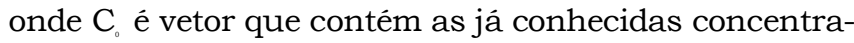
ções iniciais do cloreto de sódio e cloreto de potássio no queijo. E, uma condição de contorno específica para a salmoura com ausência de agitação [23]:

$\pm \frac{\partial C( \pm R, t)}{\partial x}=\frac{h_{m}}{\lambda_{m}}\left[C( \pm R, t)-C_{s}\right]_{\text {para }} t>0$ e $x= \pm R$

onde, $\mathrm{h}_{\mathrm{m}}\left(\mathrm{g} / \mathrm{cm}^{2} . \mathrm{h}\right)$ é o coeficiente de transferência de massa, $\lambda_{\mathrm{m}}(\mathrm{g} / \mathrm{cm} . \mathrm{h})$ é a condutividade mássica, e C é o vetor que representa a concentração dos sais na salmoura. A solução do sistema de equações formado pelas condições de contorno, condições iniciais e pela lei de Fick aplicável, inicia-se com a transformação da variável concentração empregando procedimentos clássicos de álgebra linear. O objetivo da transformação é desacoplar as equações que representam a difusão de cada soluto. A transformação de variável está baseada no teorema das matrizes semelhantes:

$\Lambda=T^{-1} D T$

onde $\Lambda$ e $\mathrm{T}$ são, respectivamente, a matriz dos autovalores e a matriz dos autovetores associados a $D$, que caso ficam sendo:

$\Lambda=\left[\begin{array}{cc}\lambda_{1} & 0 \\ 0 & \lambda_{2}\end{array}\right] \quad$ e $\quad T=\left[\begin{array}{cc}1 & -\frac{D_{22}-\lambda_{2}}{D_{21}} \\ \frac{-D_{21}}{D_{22}-\lambda_{1}} & 1\end{array}\right]$

onde cada autovalor $(\lambda)$ pode ser calculado através da equação (4), isto é,

$$
\lambda_{1,2}=\frac{D_{11}+D_{22} \pm \sqrt{\left(D_{11}-D_{22}\right)^{2}+4 D_{12} D_{21}}}{2}
$$

Definindo o vetor de concentração transformada por:

$C=T \Psi$

e substituindo em (1) vem

$\frac{\partial T \Psi}{\partial t}=D \frac{\partial^{2} T \Psi}{\partial x^{2}}$

ou ainda,

$T \frac{\partial \Psi}{\partial t}=D T \frac{\partial^{2} \Psi}{\partial x^{2}}$

Multiplicando esta equação por $T$, obtém-se:

$T^{-1} T \frac{\partial \Psi}{\partial t}=T^{-1} D T \frac{\partial^{2} \Psi}{\partial x^{2}}$

ou, levando em consideração (3), resulta

$\frac{\partial \Psi}{\partial t}=\Lambda \frac{\partial^{2} \Psi}{\partial x^{2}}$

A expressão de Fick transformada (equação 9) pode ser escrita na forma matricial e facilmente desacoplada nas equações que representam a difusão em termos das variáveis transformadas (equações 10 e 11).

$$
\frac{\partial \psi_{1}}{\partial t}=\lambda_{1} \frac{\partial^{2} \psi_{1}}{\partial x^{2}} \quad \text { (10) } \quad \frac{\partial \psi_{2}}{\partial t}=\lambda_{2} \frac{\partial^{2} \psi_{2}}{\partial x^{2}}
$$

As equações (10) e (11) podem ser resolvidas separadamente. O resultado obtido pode ser convertido para as variáveis originais através da equação (5). 
O procedimento de solução das equações transformadas (equações 10 e 11), para uma salmoura submetida à condição inicial e de contorno proposta, foi realizado segundo LUNA \& BRESSAN [23]. Para pequenos valores do número de Fourier de massa $(\mathrm{Fo}<0,1)$, ou seja, para tempos curtos, a solução aproximada pode ser descrita como:

$\frac{\psi_{i}-\psi_{i, 0}}{\psi_{i, s}-\psi_{i, 0}}=\operatorname{erfc}\left[\frac{1-\left(\frac{(}{R}\right)}{2 \sqrt{F o}}\right]-\exp \left[B i \cdot\left(1-\frac{x}{R}\right)+B i^{2} \cdot F o\right] \cdot \operatorname{erfc}\left[\frac{1-\left(\frac{(}{R}\right)}{2 \sqrt{F O}}+B i \cdot \sqrt{F O}\right]+$

$\operatorname{erfc}\left[\frac{1+(X / R}{2 \sqrt{F o}}\right)-\exp \left[B i \cdot\left(1+\frac{x}{R}\right)+B i^{2} \cdot F o\right] \cdot \operatorname{erfc}\left[\frac{1+(X / R)}{2 \sqrt{F o}}+B i \cdot \sqrt{F o}\right] \quad$ para $i=1,2$ onde $F_{O}=\frac{\lambda_{i} \cdot t}{R^{2}}$, com $\lambda$ representando os autovalores, erfc indica a função erro complementar e o número de Biot para transferência de massa (Bi) é igual a $\quad B i=\frac{h_{m} \cdot R}{\lambda_{m}}$

O número de Biot para massa pode ser interpretado como a razão entre a resistência interna e a resistência externa [9, 21]. Quanto maior Bi, menor será a influência da resistência externa sobre o mecanismo de difusão. Segundo SCHWARTZBERG \& CHAO [30], se Bi > 200, o erro relativo no coeficiente de difusão, devido ao fato de se desprezar a resistência externa, é menor do que $1 \%$.

Para a aplicação do modelo formulado foi construído um programa computacional em linguagem Fortran 90. Um fluxograma representando a seqüência matemática e computacional (algoritmo) para a simulação da difusão multicomponente através do modelo analítico (equação 12) pode ser observado na Figura 2.

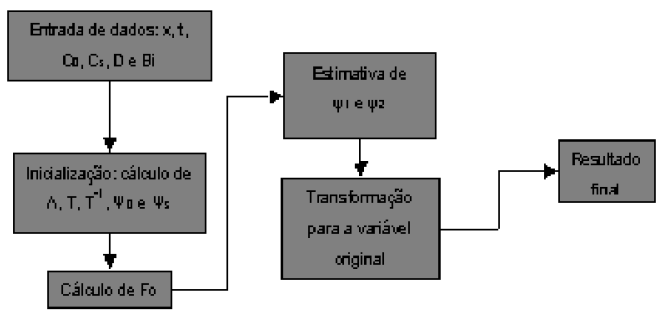

FIGURA 2 - Fluxograma que representa as etapas da simulação multicomponente através do modelo analítico (equação 12)

Os valores experimentais obtidos representam a concentração média de $\mathrm{NaCl}$ e $\mathrm{KCl}$ para a amostra cilindrica. Entretanto, o método analítico de simulação fornece concentrações pontuais. Portanto, foi necessário calcular uma concentração média para os pontos simulados sobre o eixo de amostragem. Esta média foi calculada através de uma integral de volume:

$$
\bar{C}=\frac{\int_{V} C \mathrm{~d} V}{\int_{V} \mathrm{~d} V}
$$

onde $\mathrm{C}$ é a concentração pontual calculada através do mé- todo de simulação e $\mathrm{V}$ é o volume da amostra analisada. A equação (13) foi transformada para coordenadas cilíndricas e normalizada [23]. Devido à diferença de tamanho entre o queijo e a amostra retirada, e ao fato do processo de difusão ser suficientemente lento, a penetração radial do sal na amostra pode ser desprezada e, portanto, a expressão para a concentração média se reduz para:

$$
\bar{C}=\int_{\xi=0}^{1,0} C(\xi) d \xi
$$

Esta integral foi calculada através do método de integração numérica de Gauss:

$\bar{C}=\sum_{i=1}^{m} w_{i} C\left(\xi_{i}\right)$

Os valores (Tabela 1) das coordenadas ( $\xi$ ) e dos pesos (w) foram obtidos em DAVIS \& POLONSKY [6].

TABELA 1 - Posições e pesos para a integração numérica de

\begin{tabular}{|c|c|c|c|}
\hline Ponto & Posição normalizada ( $(\xi)$ & Peso (w) & Posição real (x) \\
\hline 1 & 0,0198550718 & 0,0506142681 & 0,0794202872 \\
\hline 2 & 0,1016667613 & 0,1111905172 & 0,4066670452 \\
\hline 3 & 0,2372337950 & 0,1568533229 & 0,9489351800 \\
\hline 4 & 0,4082826788 & 0,1813418917 & 1,6331307152 \\
\hline 5 & 0,5917173212 & 0,1813418917 & 2,3668692848 \\
\hline 6 & 0,7627662050 & 0,1568533229 & 3,0510648200 \\
\hline 7 & 0,8983332387 & 0,1111905172 & 3,5933329548 \\
\hline 8 & 0,9801449282 & 0,0506142681 & 3,9205797128 \\
\hline
\end{tabular}
Gauss.

A comparação entre dados teóricos e experimentais foi feita por meio do desvio percentual [16], como forma de avaliar a qualidade do ajustamento.

$\%$ desvio $=100 \sqrt{\sum_{i=1}^{N}\left[\left(\frac{\bar{C}_{\text {calc }}-\bar{C}_{\exp }}{\bar{C}_{\exp }}\right)_{i}\right]^{2} \frac{1}{N-1}}$

onde:

$\bar{C}_{\text {calc }}=$ concentração média estimada pelo modelo analítico;

$\bar{C}_{\exp }=$ concentração experimental média;

$\mathrm{N}$ = número de observações consideradas.

Os coeficientes de difusão e o número de Biot foram ajustados através do método de otimização simplex [34] associado às funções de desejabilidade [7]. Um algoritmo de otimização [3] propôs combinações para os coeficientes de difusão e número de Biot. Estes valores foram avaliados através da formulação analítica (equação 12). As concentrações de $\mathrm{NaCl}$ e $\mathrm{KCl}$ simuladas foram então comparadas aos resultados experimentais através de um teste de avaliação estatística (equação 16). Os desvios percentuais encontrados foram avaliados pelo método de otimização que forneceu novas combinações de valores com o objetivo de minimizar o desvio entre os valores simulados e os experimentais. O procedimento foi repetido até a estabilização dos valores calculados para os desvios, coeficientes de difusão e número de Biot. 


\section{3 - RESULTADOS E DISCUSSÃO}

Para a amostra que não foi submetida à salga os valores de umidade, gordura e os teores de cloreto de sódio e cloreto de potássio determinados encontram-se na Tabela 2.

TABELA 2 - Características da amostra $\left(^{*}\right)$ de queijo prato antes de ser submetido à salga

\begin{tabular}{lc}
\hline Umidade $(\mathrm{g} / 100 \mathrm{~g}$ de queijo base & $46,2 \pm 0,2$ \\
úmida) & \\
Gordura (g/100g de queijo base seca) & $52,8 \pm 1,1$ \\
$\mathrm{NaCl}(\mathrm{g} / 100 \mathrm{~g}$ de água no queijo) & $0,178 \pm 0,001$ \\
$\mathrm{KCl}(\mathrm{g} / 100 \mathrm{~g}$ de água no queijo) & $0,275 \pm 0,004$ \\
\hline${ }^{*}$ Duplicata.
\end{tabular}

Os teores de umidade e gordura do queijo estão dentro dos padrões recomendados para o tipo prato [22]. Devido à composição média do leite [31], a concentração de cloreto de potássio no queijo antes da salga, é maior que a do cloreto de sódio.

A variação de concentração salina, em função do tempo, nas amostras pode ser observada na Tabela 3. Como suposto, a concentração salina nos cilindros retirados da face superior foi menor quando comparada à face inferior em todos os tempos de amostragem.

TABELA 3 - Variação da concentração dos sais nas amostras das faces inferior e superior em função do tempo

\begin{tabular}{ccccc}
\hline \multirow{2}{*}{$\begin{array}{c}\text { Tempo } \\
\text { (h) }\end{array}$} & $\begin{array}{c}\text { Concentração (g/100g } \\
\text { de água do queijo) }\end{array}$ & $\begin{array}{c}\text { Proporção } \\
(\%)\end{array}$ & $\begin{array}{c}\text { Concentração }(\mathrm{g} / 100 \mathrm{~g} \\
\text { de água do queijo) }\end{array}$ & $\begin{array}{c}\text { Proporção } \\
(\%)\end{array}$ \\
\hline 0 & 0,178 & 39,3 & 0,275 & 60,7 \\
1 & $0,789(0,490)$ & 67,1 & $0,387(0,334)$ & 32,9 \\
2 & $1,015(0,540)$ & 71,2 & $0,411(0,367)$ & 28,8 \\
3 & $1,116(0,567)$ & 60,3 & $0,735(0,602)$ & 39,7 \\
5 & $1,722(0,855)$ & 62,0 & $1,054(0,677)$ & 38,0 \\
7 & $2,034(1,116)$ & 63,3 & $1,178(0,845)$ & 36,7 \\
9 & $2,360(1,220)$ & 63,2 & $1,375(1,056)$ & 36,8 \\
11 & $2,526(2,027)$ & 63,9 & $1,425(1,273)$ & 36,1 \\
\hline \multicolumn{5}{l}{ Os valores entre parênteses representam a concentração das amostras da face superior. }
\end{tabular}

Com exceção dos tempos de 1, 2 e 3 horas, a proporção entres os sais manteve-se em torno de $63 \%$ de $\mathrm{NaCl}$ para $37 \%$ de $\mathrm{KCl}$. A porcentagem de cloreto de potássio obtida experimentalmente está de acordo com o valor determinado por RAPACCI [28] nas mesmas condições experimentais. Para 1 e 2 horas de salga, a concentração de cloreto de potássio determinada foi proporcionalmente inferior ao esperado. Já para 3 horas de salga a quantidade de cloreto de sódio foi inferior.

Ao final do periodo de salga, determinou-se a concentração de $\mathrm{NaCl}$ e $\mathrm{KCl}$ e o pH da salmoura, os quais mantiveram-se constantes em relação ao início da salga (Tabela 4).

Ajustes recursivos (variando-se as condições iniciais de otimização) dos coeficientes de difusão e do número de Biot, relativamente aos dados experimentais, foram realizados utilizando-se o método simplex [3]. Verificou-se, entretanto, uma instabilidade e falta de convergência das respostas. A partir dos dados experimentais e da pro- porção entre os sais em cada tempo amostral, observouse que a concentração experimental obtida para o $\mathrm{NaCl}$ em 3 horas de salga, e as concentrações em 1 e 2 horas de salga para o $\mathrm{KCl}$ apresentaram um comportamento anômalo em relação aos demais pontos que compõem o perfil experimental de cada sal. Baseado nesta constatação, estes pontos não foram utilizados no ajuste dos coeficientes de difusão e do número de Biot. A partir daí, as novas tentativas de otimização apresentaram convergência e estabilização relativamente rápida para as respostas (desvios em relação aos dados experimentais do $\mathrm{NaCl}$ e $\mathrm{KCl}$ ) e variáveis ajustadas (coeficientes de difusão e número de Biot), como pode ser visto nas Figuras 3 e 4 .

TABELA 4 - Características iniciais da salmoura utilizada para a salga mista do queijo prato

\begin{tabular}{lc}
\hline Temperatura durante a salga $\left({ }^{\circ} \mathrm{C}\right)$ & $10 \pm 1$ \\
$\mathrm{pH}$ & 5,6 \\
$\mathrm{CaCl}_{2}(\% \mathrm{~m} / \mathrm{m})$ & 0,5 \\
$\mathrm{NaCl}(\% \mathrm{~m} / \mathrm{m})$ & 15,0 \\
$\mathrm{KCl}(\% \mathrm{~m} / \mathrm{m})$ & 5,6 \\
Proporção entre os sais & $72,8 \% \mathrm{de} \mathrm{NaCl}$ \\
& para $27,2 \% \mathrm{de} \mathrm{KCl}$ \\
Relação entre o volume de salmoura e o volume de queijo & 3,5 \\
\hline *
\end{tabular}
* As condições finais da salmoura são análogas às iniciais.

Os desvios foram estabilizados, após cerca de 30 interações do algoritmo de otimização (minimização), em $2,7 \%$ para o $\mathrm{NaCl}$ e 6,6\% para o $\mathrm{KCl}$. Já a estabilização das variáveis só foi possivel após 55 interações. As estimativas para os coeficientes de difusão e o número de Biot encontram-se na Tabela 5.

TABELA 5 - Valores ajustados para os parâmetros do modelo analítico

\begin{tabular}{lll}
\hline & $\mathrm{NaCl}$ & \multicolumn{1}{c}{$\mathrm{KCl}$} \\
\cline { 2 - 3 } $\begin{array}{l}\text { Coeficientes principais } \\
\left(\mathrm{cm}^{2} / \text { dia }\right)\end{array}$ & $0,225\left(\mathrm{D}_{\mathrm{t}}\right)$ & $0,240\left(\mathrm{D}_{22}\right)$ \\
Coeficientes cruzados $\left(\mathrm{cm}^{2} /\right.$ dia $)$ & $0,027\left(\mathrm{D}_{\mathrm{i} 2}\right)$ & $0,045\left(\mathrm{D}_{2,}\right)$ \\
\hline Número de Biot (adimensional) & & 40,660 \\
$\mathrm{~h}_{\mathrm{m}} \lambda_{\mathrm{m}}(\mathrm{cm})$ & & 20,330 \\
\hline
\end{tabular}

Em relação aos coeficientes de difusão principais ajustados, $\mathrm{D}_{1}$, é menor do que $\mathrm{D}_{22}$, o que está de acordo com a literatura consultada, que estima uma maior mobilidade do potássio na água [30]. Além disso, o fato da proporção de cloreto de potássio na salmoura (Tabela 4) ser menor que no queijo sugere que a velocidade de migração do cloreto de potássio seja maior que a do cloreto de sódio.

Os coeficientes de difusão cruzados ajustados apresentam uma ordem de grandeza inferior aos coeficientes principais indicando como era esperado, que a difusão em relação ao próprio gradiente é mais importante do que a difusão devida à interação entre os solutos [20, 32]. Entretanto, estes coeficientes são necessários para um bom ajuste entre os dados experimentais e o modelo teórico. O perfil experimental e o calculado pelo modelo 

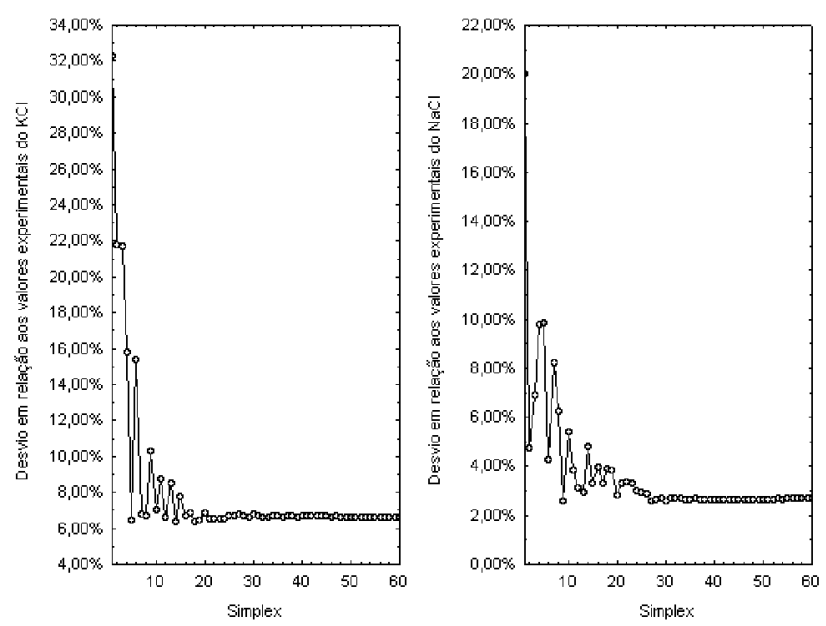

FIGURA 3 - Estabilização dos desvios em relação aos dados experimentais
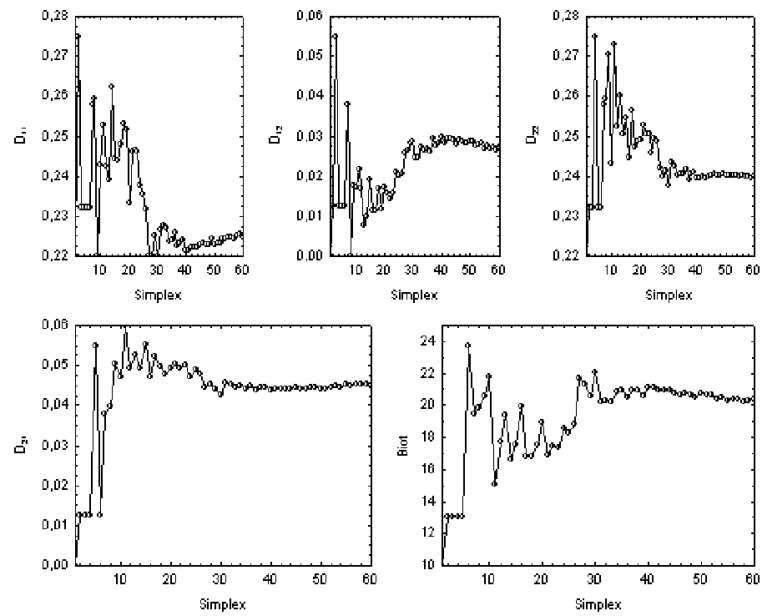

FIGURA 4 - Estabilização dos parâmetros estimados através do método simplex

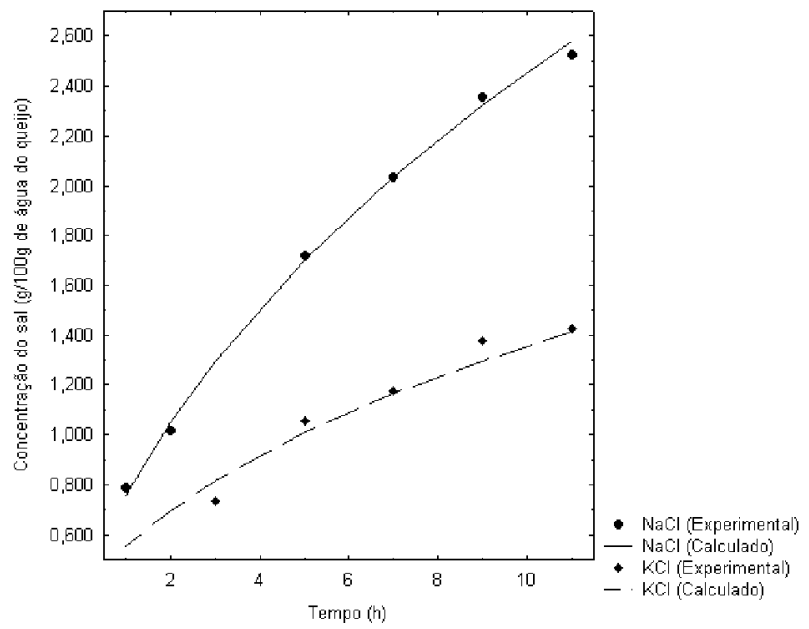

FIGURA 5 - Valores experimentais e estimados através do modelo teórico (equação 12) (equação 10) podem ser vistos na Figura 5, onde se observa o ajuste satisfatório do modelo aos dados experimentais.

Com relação ao número de Biot (Tabela 5), conclui-se que a resistência externa é importante na transferência de massa estudada [30] e deve ser considerada quando a salga for realizada em salmouras estáticas.

\section{4 - CONCLUSÕES}

- Foi possível modelar a difusão multicomponente durante a salga de queijo prato através da segunda lei de Fick generalizada para dois solutos ( $\mathrm{NaCl}$ e $\mathrm{KCl}$ ).

A modelagem analítica unidimensional empregada foi eficaz para a estimativa dos coeficientes de difusão e o número de Biot.

- As estimativas para os coeficientes de difusão indicaram que a interação entre os solutos é menos importante que a difusão isolada de cada soluto. Entretanto, devem ser considerados para uma melhor concordância do modelo aos valores experimentais.

A estimativa do número de Biot revelou que a resistência externa deve ser considerada no modelo quando a salga é realizada sem agitação.

- O modelo teórico (equação 12) possibilitou a simulação da difusão durante a salga, mostrando-se uma ferramenta promissora para o controle de qualidade durante a fabricação de queijos salgados por imersão. Concomitantemente, a modulação dos teores salinos enseja uma possivel redução no tempo de salga, com ganhos evidentes de produtividade. Além dos benefícios supracitados, um modelo bem ajustado ao sistema em questão pode ser utilizado para a previsão das características do produto quando mudanças nas variáveis do processo forem implementadas. Registre-se, portanto, a importância do desenvolvimento de modelos que simulem sistemas reais para ensejar a otimização e o controle de qualidade em processos agroindustriais.

\section{5 - REFERÊNCIAS BIBLIOGRÁFICAS}

[1] ABERNETHY, J. D. M. D. Sodium and potassium in high blood pressure. Food Technology, v.33, n.12, p.57-59, 1979.

[2] AOAC Association of Official Agricultural Chemists. Official Methods of Analysis, 14 ed.,1984. 1141p.

[3] BONA, E.; BORSATO, D.; SILVA, R. S. S. F.; HERRERA, R. P. Aplicativo para otimização empregando o método simplex seqüencial. Acta Scientiarum, v.22, n.5, p.1201-1206, 2000.

[4] CRANK, J. The Mathematics of Diffusion. London: Oxford University Press, 1975, 414p.

[5] CUSSLER, E. L. Multicomponent Diffusion. Amsterdan: Elsevier, 1976, 176p.

[6] DAVIS, P. J.; POLONSKY, I. Numerical interpolation, differentiation and integration. In: ABRAMOWITZ, M. and STEGUN, I. (ed.) Handbook of Mathematical Functions, New York: Dover Publications, cap. 25, 
p.875-924, 1965

[7] DERRINGER, G.; SUICH, R. Simultaneous optimization of several response variables. Journal of Quality Technology, v.12, n.4, p.214-219, 1980.

[8] FITZGERALD, E.; BUCKLEY, J. Effect of total and partial substituition of sodium chloride on the quality of cheddar cheese. Journal of Dairy Science, v.68, n.12, p.31273134, 1985.

[9] FRYER, P.J.; PYLE, D. L.; RIELly, C. D. Chemical Engineering for the Food Industry. London: Blackie Academic \& Professional, 1997. 462p.

[10] FURTADO, M. M. A arte e a ciência do queijo. São Paulo: Editora Globo S.A., 1990, 297p.

[11] FURTADO, M. M.; SOUZA, H. M. Estudo rápido sobre a evolução da salga do queijo prato em salmoura. Revista ILCT, jan-fev, p.5-10, 1981.

[12] GERLA, P. E.; RUBIOLO, A. C. A model for determination of multicomponent diffusion coefficients in foods. Journal of Food Engineering, v.56, p.401-410, 2003.

[13] GUINNE, T. P.; FOX, P. F. Salt in cheese: physical, chemical and biological aspects. In: FOX, P. F. (ed.) Cheese: chemistry, physics and microbiology. New York: Elsevier Applied Publ. Science, v. 1, p.31-35, 1984.

[14] HE, F. J.; MACGREGOR, G. A. Potassium intake and blood pressure. American Journal of Hypertension, v.12, p.849-851, 1999.

[15] HE, F. J.; MACGREGOR, G. A. Benefical effects of potassium. British Medical Journal, v.323, p.497-501, 2001.

[16] HELDMAN, D. R. Predicting the relationships between unfrozen water fraction and temperature during food freezing using freezing point depression. Transactions ASAE, v. 17, p.63, 1974.

[17] JAMESON, G. W. Dietary cheese: low fat, low salt. CSIRO Food Research Journal, v.46, p.64, 1986.

[18] KATSIARI, M. C.; VOUTSINAS L. P.; ALICHANIDIS, E.; ROUSSIS, I. G. Manufacture of kefalograviera cheese with less sodium by partial replacement of $\mathrm{NaCl}$ with $\mathrm{KCl}$. Food Chemistry, v.61, n.1/2, 0.63-70, 1998.

[19] LINDSAY, R. C.; HARGETT, S. M.; BUSH, C. S. Effect of sodium/potassium (1:1) chloride concentrations on quality of cheddar cheese. Journal of Dairy Science, v.65, n. 3, 1982

[20] LOMBARDI, A. M.; ZARITZKY, N. E. Simultaneous diffusion of citric acid and ascorbic acid in prepeeled potatoes. Journal os Food Process Engineering, v.19, p.27-48, 1996.

[21] LONCIN, M.; MERSON, R. L. Food Engineering: principles and selected applications. London: Academic Press, 1979. 494p.
[22] LONQUE, H.; ANTUNES, L. A. F. Emprego de culturas nativas na fabricação de queijo prato. II. Características do produto. Arq. Biol. Tecnol., Curitiba, v.33, p.561573, 1990.

[23] LUNA, J. A.; BRESSAN, J. A. Mass transfer during brining of cuartirolo argentino cheese. Journal of Food Science, v.51, n.3, p.829-831, 1986.

[24] LYNCH, N. In search of the salty taste. Food Technology, v.41, n.11, p.82-86, 1987.

[25] MEDINA-VIVANCO, M.; SOBRAL, P. J. A.; HUBINGER, M. D. Osmotic dehydration of tilapia fillets in limited volume of ternary solutions. Chemical Engineering Journal, v.86, p.199- 205, 2002.

[26] ONSAGER, L. Theories and problems of liquid diffusion. Ann. N. Y. Acad. Sci., v.46, p.241, 1945.

[27] PAYNE, M. R.; MORISON, K. R. A multi-component approach to salt and water diffusion in cheese. International Dairy Journal, v.9, p.887-894, 1999.

[28] RAPACCI, M. Efeito da substituição do cloreto de sódio por cloreto de potássio nas características do queijo prato. Londrina, 1989, 106p. Tese de Mestrado. Universidade Estadual de Londrina.

[29] SILVA, R. S. F.; BORSATO, D.; SILVA, L. H. M. Difusão do cloreto de sódio no processo de salga de queijos: modelagem matemática com o emprego do método de elementos finitos. Ciênc. Tecnol. Aliment., v.18, n.1, p87-92, 1998.

[30] SCHWARTZBERG, H. G.; CHAO, R. Y. Solute diffusivities in leaching processes. Food Technology, v.36, n.2, p.7386, 1982.

[31] SWAISGOOD, H. E. Characteristics of Milk. In: FENNEMA, O. R. (ed.) Food Chemistry. New York: Marcel Dekker, 3 ed., 1996, cap. 14, p. 842-876.

[32] ZORRILLA, S. E.; RUBIOLO, A. C. A model for using the diffusion cel in the determination of multicomponent diffusion coefficients in gels or food. Chemical Engineering Science, v.49, n.13, p.2123-2128, 1994.

[33] ZORRILlA, S. E.; RUBIOLO, A. C. Modelling average concentrations of salt and salt substitute in partial or total volumes of semihard cylindrical cheeses. International Journal of Food Science and Technology, v.33, p.501-508, 1998.

[34] WALTERS, F. H.; MORGAN, S. L.; PARKER, L. R.; DEMING, S. N. Sequential simplex optimization. Karskrona, Sweden: Licensed eletronic reprint by Multisimplex AB, 1999, 403p.

\section{6 - AGRADECIMENTOS}

À Fundação Araucária, pelo auxílio financeiro para a compra dos materiais e reagentes, e à CAPES, pela concessão de bolsa de mestrado. 